\title{
Extracapsular subcutaneous endoscopic treatment for refractory lateral epicondylalgia: technique, retrospective results, and prognostic factors
}

\author{
Yu-Ping Yang", Shuo Yuan", Guo-Qing Cui, Ning An, Ying-Fang Ao \\ Institute of Sports Medicine, Peking University Third Hospital, Beijing, China \\ Contributions: (I) Conception and design: YF Ao, YP Yang; (II) Administrative support: YF Ao, GQ Cui; (III) Provision of study materials or patients: \\ YP Yang; (IV) Collection and assembly of data: S Yuan, N An; (V) Data analysis and interpretation: S Yuan; (VI) Manuscript writing: All authors; (VII) \\ Final approval of manuscript: All authors. \\ \#These authors contributed equally to this work. \\ Correspondence to: Ying-Fang Ao. 49 North Garden Rd., Haidian District, Beijing, China. Email: yingfang.ao@vip.sina.com.
}

Background: The extracapsular subcutaneous endoscopic treatment is a new and under-researched
approach to the endoscopic treatment of refractory lateral epicondylalgia. We aimed to introduce the
techniques of the method and the mid-long term clinical effects. Furthermore, we intended to identify
demographic and surgical-related factors correlated with prognosis of extracapsular subcutaneous method.
Methods: Patients with a minimum of 6 months of conservative treatment for lateral epicondylalgia were
recruited from March 2012 to July 2018. Key surgical techniques including microfracture and endoscopic
suture were used. Visual analog scale (VAS), Mayo Elbow Performance Score (MEPS), and Disability of
Arm (Shoulder and Hand, DASH) scores were used to evaluate clinical results. Univariable analysis and
multivariable logistic regression were used to analyze effects of demographic characteristics and treatment
techniques on prognosis.

Results: Seventy-eight patients were treated with modified subcutaneous extracapsular surgery. No serious complications such as neurovascular injury or infection occurred. Postoperative VAS, MEPS, and DASH scores significantly improved $(\mathrm{P}<0.001)$. Age was associated with prognosis regarding VAS score $(\mathrm{P}=0.023)$. Older age was an independent relevant factor for poor prognosis regarding VAS score (OR $=0.914,95 \%$ CI: 0.842-0.993, $\mathrm{P}=0.033$ ), and microfracture during surgery related to poor prognosis for DASH score (OR $=0.056,95 \%$ CI: $0.004-0.783, \mathrm{P}=0.032)$. Plaster fixation was an independent factor related to good prognosis regarding DASH score (OR $=11.156,95 \%$ CI: 1.009-123.363, $\mathrm{P}=0.049)$.

Conclusions: Extracapsular subcutaneous method of refractory lateral epicondylalgia has satisfactory and stable clinical results with high safety. Techniques of extracapsular method include debridement, microfracture, and tendon suture under endoscope. Young age, no microfracture, and postoperative plaster fixation were associated with good long-term prognosis.

Keywords: Refractory lateral epicondylalgia (RLE); endoscopy; intracapsular method; extracapsular method; prognostic factors

Submitted Oct 08, 2020. Accepted for publication Feb 26, 2021.

doi: 10.21037/atm-20-6799

View this article at: http://dx.doi.org/10.21037/atm-20-6799

\section{Introduction}

Refractory lateral epicondylalgia (RLE), which usually refers to lateral epicondylalgia that is not sufficiently relieved after 6 months of intensive conservative treatment, is generally considered a surgical indication (1). Compared with traditional open surgery, arthroscopic treatment is 

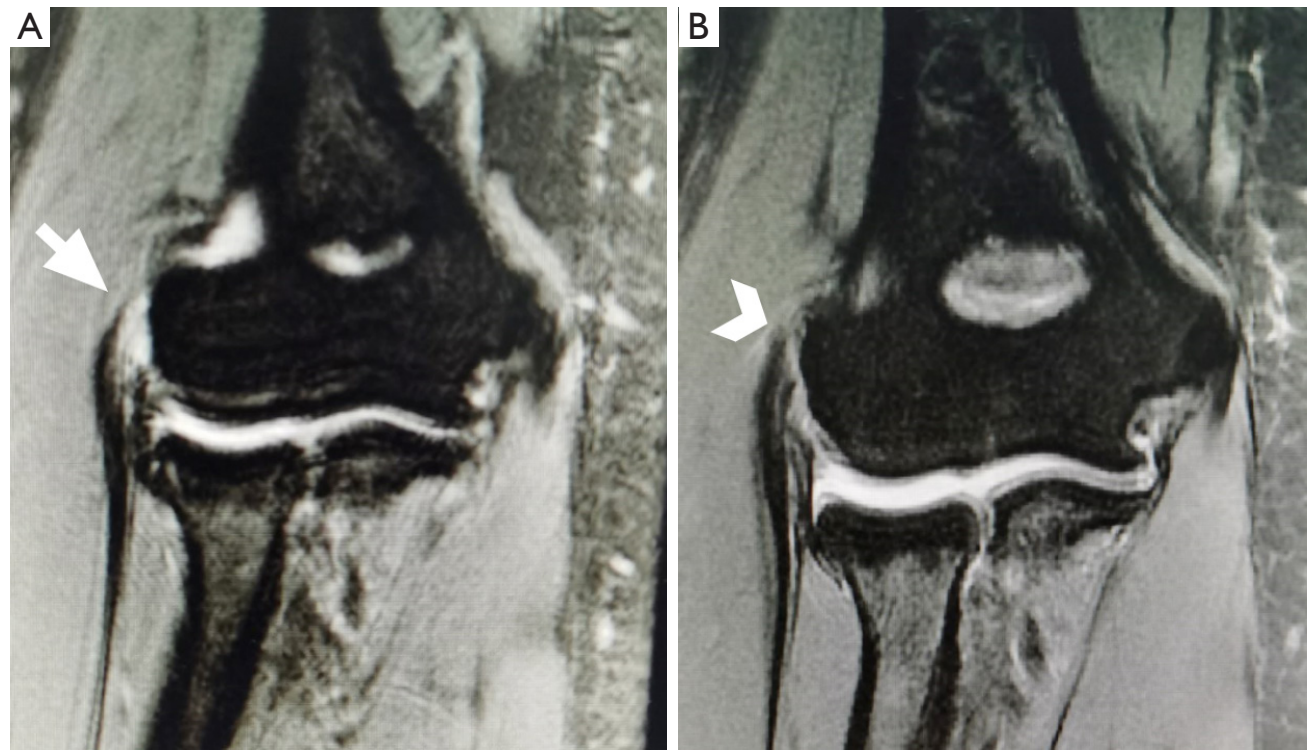

Figure 1 Preoperative and 6-month postoperative coronal proton density-weighted fat-saturated MR images obtained in a 46-year-old woman. (A) A region of increased signal intensity due to fluid accumulation at the site of the expected ECRB tendon origin (arrow); a small partial-thickness tear of the CEO can also be noticed. (B) Fluid accumulation was significantly decreased 6 months after surgery; no obvious tear was found (arrowhead).

gaining popularity with potential advantages including less trauma to the patient, better inspection of tendon structure, and lower probability of iatrogenic morbidity $(2,3)$. Currently, most arthroscopic procedures require elbow capsule resection and entrance to joint cavity (referred to as the "intracapsular method"), which causes damage to the joint capsule and risks intraarticular neurovascular injury $(4,5)$. Moreover, the view under arthroscopy is not ideal because the capitulum obstructs the view of the common extensor origin (CEO) (6). Because of these disadvantages, researchers (7-10) explored the "extracapsular method", an endoscopic approach that employs release or debridement techniques in subcutaneous working space outside the joint capsule instead of in articular cavity.

In 2005, we launched a modified extracapsular method for the treatment of RTE. The method had good results in a small-sample retrospective study $(10,11)$. The present study aimed to introduce the modified subcutaneous extracapsular surgery and clinical effects in a mid-long term follow-up. Furthermore, the prognostic factors of surgical treatment for RLE, including from demographic to surgical-related factors, are still under research (12-14). Also, there is lack of evidence suggesting that certain operation technique can improve the results of extracapsular method. We intended to identify demographic and surgical-related factors that correlate with prognosis, including age, sex, glucorticoid injection, preoperative condition and surgical techniques including microfracture and suture. We hypothesized that the extracapsular method significantly releases pain and improves function of patients with RLE, and it would result in low rate of complications. We also hypothesized that certain techniques are helpful in the prognosis of these patients. We present the following article in accordance with the STROBE reporting checklist (available at http:// dx.doi.org/10.21037/atm-20-6799).

\section{Methods}

\section{Patients sample}

The study included consecutive patients who underwent modified extracapsular surgery by single surgeon practice in the Institute of Sports Medicine, Peking University Third Hospital from March 2012 to July 2018. Inclusion criteria were history of chronic activity-related lateral elbow pain that hampered the activity of daily living (ADL), significant signs at physical examination including tenderness at the origin of extensor and pain with resisted wrist extension, obvious degeneration or tearing of the CEO in MRI examination (Figure 1), and failure of conservative treatment no less than 6 months. Exclusive criteria included history 

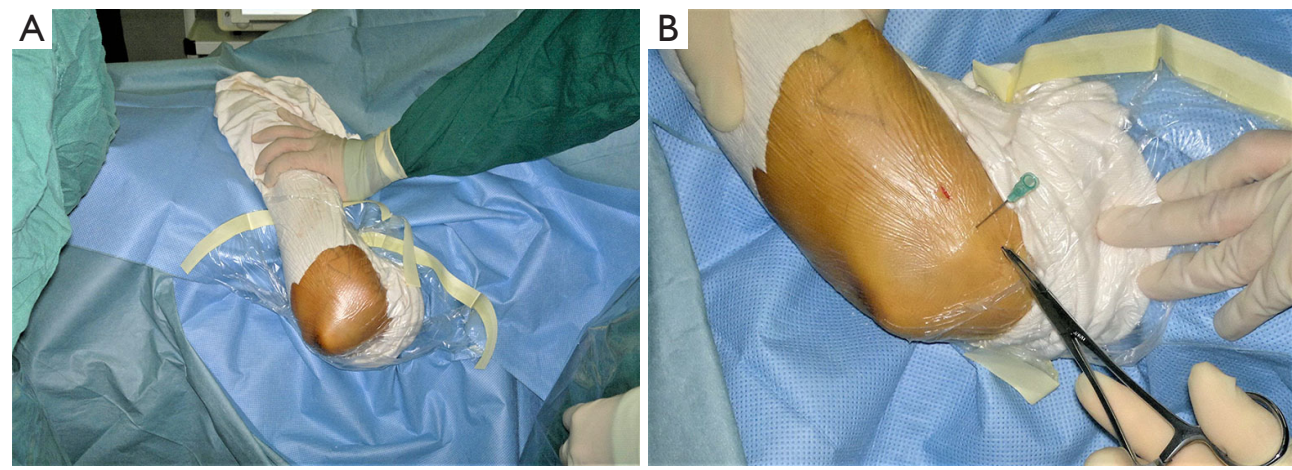

Figure 2 Patient position and location of incisions. (A) Supine position was adopted for the extracapsular method; the affected elbow was placed on the chest with a gas tourniquet. (B) The lateral epicondyle was probed with a needle, and two incisions (0.5 cm in length) were made $3 \mathrm{~cm}$ proximal and distal of the lateral epicondyle.

of elbow surgery, other elbow lesions, and RTE combined with nerve or blood vessel injury. All participants were not involved in the design, or conduct, or dissemination plans of the study. A brief summary of the result will be distributed to every participant. The study was conducted in accordance with the Declaration of Helsinki (as revised in 2013). The study was approved by Peking University Third Hospital Ethics Review Board (No.: 2018-219-011) and informed consent was taken from all individual participants.

\section{Surgical technique}

The patient was under brachial plexus anesthesia in the supine position (Figure $2 A$ ). The affected elbow was placed on the chest with a gas tourniquet. No side table or assistant frame was required. The lateral epicondyle was probed with a needle, and two incisions $(0.5 \mathrm{~cm}$ in length) were made 3 $\mathrm{cm}$ proximal and distal of the lateral epicondyle (Figure $2 B$ ). The approach extended through the subcutaneous space to the surface of the CEO by blunt dissection. Saline solution was injected to fill the subcutaneous space. The endoscope entered from the proximal approach and the blade from the distal. Key procedures were as follows:

The surgeon cleaned and exposed the aponeurosis of the CEO by using a blade. According to the principle of the "scratch test" $(11,15)$ combined with recent MRI results before surgery, the surgeon sought tendon degeneration, weakness, or broken parts while scratching off the pathological tendon tissue to achieve thorough debridement.

Where the cortical bone of the tendon attachment point was completely exposed after debridement, the microfracture technique was performed on the cortical bone with a microfracture device after decorticalization. If cortical bone was not fully exposed, microfracture treatment was not implemented.

After the first two procedures were completed, if the joint capsule had a tear (Figure 3A), the surgeon used PDS suture (Ethicon, Cincinnati, Ohio, USA) to close the tear under endoscopy. 3-0 antibacterial Vicryl absorbable suture (Ethicon) was used to tie and strengthen the stitch (Figure 3B). Those without obvious tear on the joint capsule were not sutured.

Intracapsular ascertainment can be performed if necessary [In the present study, pathological biopsy tissue specimens were sent fresh for pathologic analysis (Figure 4)].

\section{Postoperative rebabilitation}

The elbow joint was fixed in the extended position for 2 weeks after surgery, and elbow flexion and extension gradually started after the fixation was removed. However, periarthritis symptoms appeared at the affected side 20 to 30 days after operation in certain cases, which seriously affected the postoperative rehabilitation process and surgical effect. Based on the results of a related study (16), rest of the cases were fixed with cotton bandage and splints after surgery instead of plaster cast to ensure that patients could do ADL within pain tolerance levels. After 2 weeks, fixation was removed, and a mild wrist extension exercise was started. Wrist extension exercise with normal resistance and heavy manual work were not allowed until 6 weeks after surgery. Recreational sports activities involving the affected upper limb could be started 3 months after surgery. 

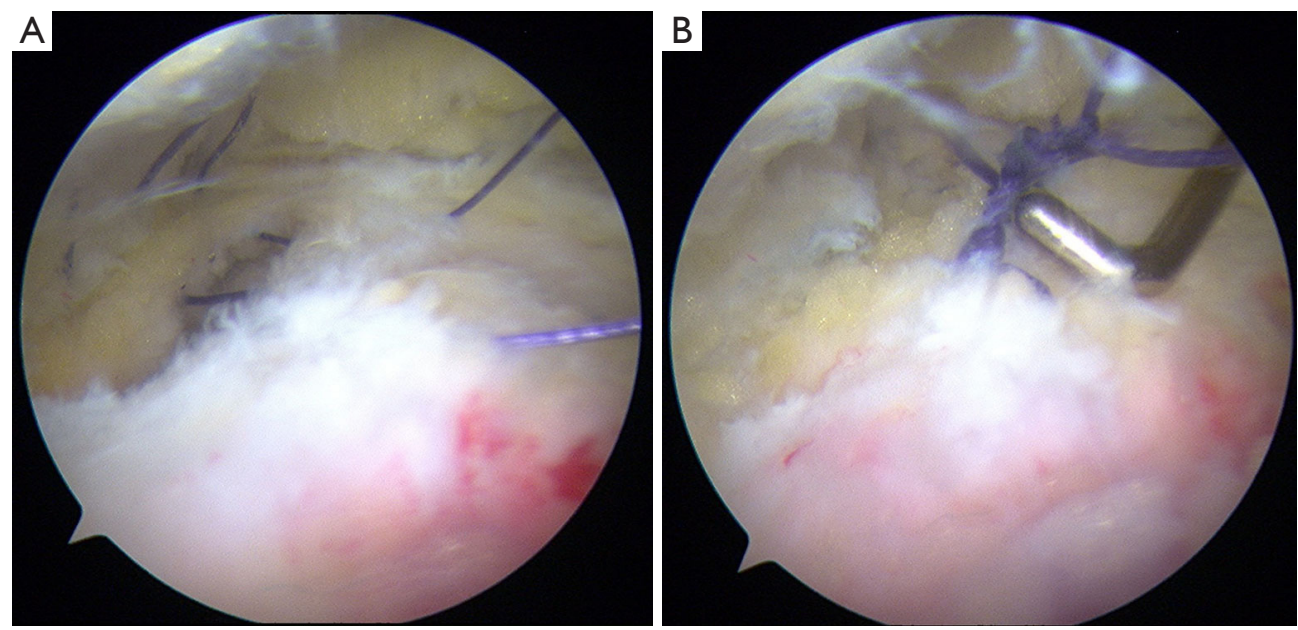

Figure 3 Tendon tear suture operation. (A) An obvious tendon tear. (B) Tear was sutured under endoscopy using PDS suture and 3-0 antibacterial Vicryl absorbable suture.
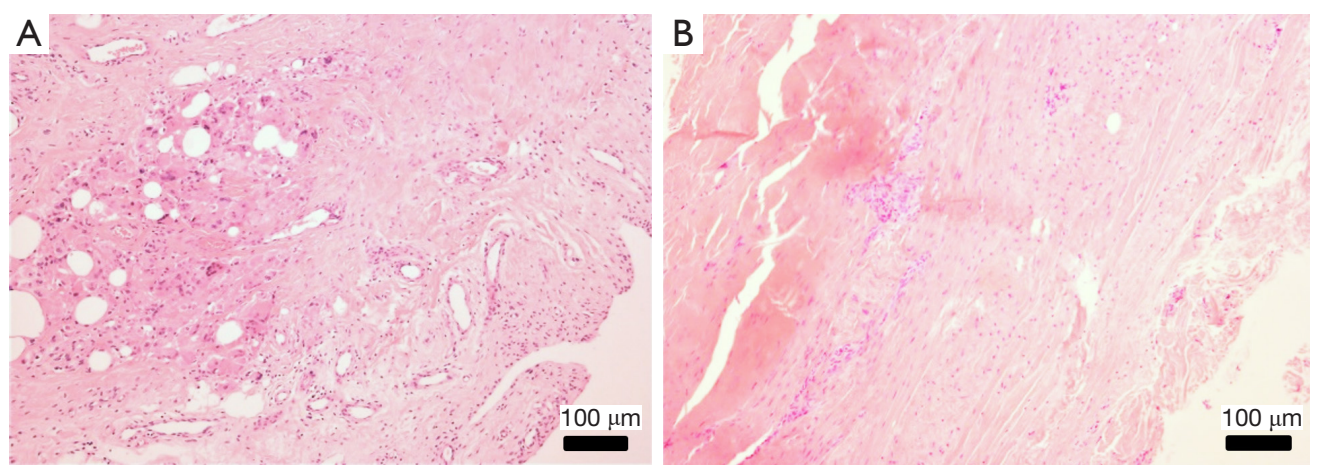

Figure 4 Pathological analysis of degenerative tendon tissue. (A) Synovial tissue. Interstitial connective tissue hyperplasia, focal vascular proliferation, focal necrosis with foam cell formation, and foreign body giant cell response. (B) Tissue from extensor carpi radialis brevis. Dense connective tissue and small vessel hyperplasia with a small amount of chronic inflammatory cell infiltration. Hematoxylin-eosin staining, magnification: $\times 10$.

\section{Outcome assessment}

Outpatient follow-up was performed at 6 weeks, 3 months, 6 months, and 12 months after the operation. The surgeon was responsible for physical examination and postoperative rehabilitation guidance during follow-up. Patients-reported outcome measures was performed by an assistant who did not participate in patient treatment. Telephone follow-up was performed by the assistant at 24 months after surgery and at the last follow-up (December 2017 to July 2019). Fifteen patients $(20 \%)$ who could not access outpatient follow-up also received the telephone follow-up from the assistant.

Physical examination in outpatient follow-up included a lateral epicondylitis tenderness test, provoked pain with resisted wrist test, and Visual Analog Scale (VAS) scores. MRI examination was performed at 6 months or 1 year after operation (Figure 1B). Patients-reported outcome measures were used for postoperative recovery evaluation: VAS scores for the degree of pain (0 points for no pain and 10 points for unbearable pain), Mayo Elbow Performance Score (MEPS) and Disability of Arm, Shoulder and Hand Questionnaire (DASH) for elbow function recovery (17). All the three measures were widely used in previous studies and have been shown to be reliable, responsive, and valid measures of upper extremity disability (18-20). ADL recovery time, work recovery time, exercise recovery time, and surgical 
Table 1 Baseline characteristics of study population $(n=75)$

\begin{tabular}{ll}
\hline Baseline characteristics & Study population $(\mathrm{n}=75)$ \\
\hline No. of Elbows & 75 \\
Age $(\mathrm{yr})$ & $45.4(\mathrm{SD}, 6.4)$ \\
Women & $43(57.3 \%)$ \\
BMl & $24.1(\mathrm{SD}, 3.3)$ \\
Duration $(\mathrm{mo})$ & $16.2(13.1,22.5)$ \\
Right Elbow & $53(70.7 \%)$ \\
Dominant Elbow & $38(50.7 \%)$ \\
History of trauma & $15(20 \%)$ \\
History of glucocorticoid injection & $65(86.7 \%)$ \\
\hline
\end{tabular}

satisfaction were recorded. Surgical satisfaction was rated using three levels: very satisfied, satisfied, and unsatisfied. In the analysis of prognosis factors, to meet the requirement of the statistical method, we transformed the continuous measures into two-category variables: complete recovery (VAS $=0$, MEPS $=100$, and DASH $=0$ at the last follow-up) and incomplete recovery (VAS $>0$, MEPS $<100$, or DASH $>0$ at the last follow-up).

\section{Statistical analysis}

Statistical analysis was conducted using SPSS 20.0 (SPSS, Chicago, IL, USA). The measurement data were tested using the Kolmogorov-Smirnov test. Data that meet the normal distribution are represented by mean (standard deviation), and those that do not are represented by median (inter-quartile range). Because some data of three measures are not normally distributed, the Wilcoxon test was used to compare differences between the three measures before and after the operation. Univariable analysis aims to evaluate the influence of 7 prognostic factors on treatment results (represented by complete recovery by VAS, MEPS, and DASH), including demographic factors (sex and age), preoperative-related factors (preoperative scores and numbers of glucocorticoid injection), technique-related factors (microfracture and tear suture), and a rehabilitationrelated factor (plaster cast fixation). For continuous variables, different statistical tests were adopted according to normality test. Age, preoperative MEPS, preoperative VAS and preoperative DASH are normally distributed, and the independent sample $t$ test was used to compare the variables between different outcomes. Numbers of glucocorticoid injections are not normally distributed, and the Mann-Whitney $U$ test was used to compare the differences between different outcomes. For the twocategory variables, the Pearson $\chi^{2}$ test or the continuous correction $\chi^{2}$ test was adopted according to the expected value of the four-fold table. Multivariate logistic regression was used to analyze the independent contributions of these factors to the likelihood of complete recovery according to the three measures. Bilateral $\mathrm{P}<0.05$ was considered statistically significant.

\section{Results}

The clinical materials of consecutive 78 patients with RLE who met the required criteria were collected. Three cases were lost. Thus, 75 cases, 32 men (42.7\%) and 43 women $(57.3 \%)$, with a mean age of 45.4 (6.4) years, were included. The baseline characteristics of the cohort are presented in Table 1. The mean BMI was 24.1 (3.3). The median conservative treatment duration was $16[13,22]$ months. There were 22 cases $(29.3 \%)$ of left elbow and 53 cases (70.7\%) of right elbow. In all, 38 cases $(50.7 \%)$ were of the dominant side. A total of 15 (20\%) patients had a history of trauma, and $65(86.7 \%)$ had a history of local glucocorticoid injection.

In all, 33 cases (44\%) were without elbow flexion or extension pain, 25 (33.3\%) were with elbow hyperextension pain, and 17 (22.7\%) were with elbow hyperextension pain; 20 cases $(26.7 \%)$ were with elbow restricted extension, whereas 55 were without elbow restricted extension. The median wrist extension VAS score was $3.0(1.0,6.0)$. Only three patients had restricted wrist flexion: the restriction angles were $5^{\circ}, 5^{\circ}$, and $35^{\circ}$, respectively; their VAS scores were 5,5 , and 8 , respectively. All cases had CEO origin degeneration of varying degree; seven had tears at CEO origin. According to the MRI classification described by Walz et al. (21), 23 cases (30.7\%) were grade 1, 34 (45.3\%) were grade 2, and 18 (24\%) were grade 3 .

\section{Effectiveness and safety of extracapsular method}

The median operation time was $41[32,55]$ minutes. Thirteen $(17.3 \%)$ cases received microfracture treatment during the procedure, 34 (45.3\%) cases received joint capsule suture during the procedure, and $21(28 \%)$ cases received elbow joint fixation after surgery. No complications such as infection or nerve injury occurred. The mean follow-up period was 31.3 (14.2) months; 44 patients were 
Table 2 Comparison of VAS, MEPS and DASH score between preoperative and last follow-up [n=75, mean (quartile 1 Q1, quartile 3 Q3)]

\begin{tabular}{lccc}
\hline & VAS & MEPS & DASH \\
\hline Preoperative & $7.0(7.0,7.0)$ & $60.0(47.0,68.0)$ & $44.2(29.2,65.0)$ \\
Last follow-up & $0.0(0.0,1.0)$ & $100(99.0,100.0)$ & $0.13(0.0,0.8)$ \\
Z value & -7.493 & -7.478 & -7.525 \\
P value & 0.000 & 0.000 & 0.000 \\
\hline
\end{tabular}

followed up for no more than 36 months, 26 patients were followed up for more than 36 months, and 5 were followed up for more than 60 months. At the last follow-up, VAS, MEPS, and DASH scores were all significantly improved $(\mathrm{P}<0.001)$ (Table 2). MEPS improvement was excellent for all patients.

At the last follow-up, three cases were positive for provoked pain with resisted wrist test (positive rate: $4 \%)$. A total of 22 cases $(29.3 \%)$ had a negative result for provoked pain with resisted wrist test but exhibited lateral epicondyle tenderness. Pain completely disappeared in 50 cases $(66.6 \%)$.

After the postoperative anesthesia faded, the tenderness of the preoperative pain site was tested, and subjective tenderness had completely disappeared in 25 patients. No pain recurred during follow-up in these 25 patients, and the final follow-up VAS score remained 0 . The median VAS score for the other 50 patients at 6 months was 3 [1, $3]$, which was significantly lower than the preoperative median VAS score of 7 [7, 8] $(Z=-7.256, P<0.001)$. Among these 50, 27 had complete recovery at the last follow-up (VAS $=0$ ), and the other 23 reported varying degrees of pain (VAS score from 1 to 6), but no significant effect on ADL was reported. VAS scores were reduced by between 1 and 9 compared with before surgery.

The ADL of all patients recovered to normal after operation, and the median recovery time was 4 weeks (3.0, 9.0). Regarding surgical satisfaction, 71 patients were very satisfied and 4 were satisfied. The four patients who were not satisfied had a postoperative VAS score of higher than 4 . Although pain was reduced compared with the preoperative pain, pain still existed, which was more obvious during heavy physical labor.

\section{Analysis of prognostic factors}

The results of prognostic factors analysis are presented in Table 3. Univariate analysis indicated that age was associated with the prognosis regarding postoperative VAS score $(\mathrm{t}=-2.327, \mathrm{P}=0.023)$. Older patients had higher risks of poor prognosis regarding pain recovery. Multivariate analysis indicated that younger age was an independent relevant factor for good prognosis of complete recovery (by VAS) after surgery $(\mathrm{OR}=0.914,95 \% \mathrm{CI}: 0.842-0.993, \mathrm{P}=0.033)$, and the absence of microfracture during surgery was a factor for good prognosis of complete recovery (by DASH) after surgery $(\mathrm{OR}=0.056,95 \% \mathrm{CI}$ : 0.004-0.783, $\mathrm{P}=0.032)$. Postoperative plaster cast fixation was also an independent relevant factor for good prognosis regarding DASH score (OR $=11.156,95 \%$ CI: $1.009-123.363, \mathrm{P}=0.049)$.

\section{Discussion}

Although previous studies (7-10) have introduced and explored the extracapsular method, the clinical efficacy and safety of the method still need to be tested in study with larger cohort. In our 75-cases study, extracapsular method significantly improved pain and function measures, and postoperative satisfaction rates were high $(95 \%$ very satisfied rate, $100 \%$ satisfied rate). No complications such as infection or nerve injury occurred after operation. When the results are combined with those of our previous research $(10,11)$, they provide strong evidence of the effectiveness and safety of extracapsular endoscopic treatment for RLE.

Some studies compared the clinical outcomes among different methods of operative treatment, including open surgery, arthroscopic treatment and percutaneous surgery. Szabo et al. (16) evaluated all the three methods and found no difference among the effects of three methods. Clark et al. (22) conducted a randomized trial to compare the effects of arthroscopic treatment versus open surgery, and the results were also negative. So far, there is no casecontrol study comparing extracapsular study with other operative treatment. High-quality RCTs are needed to optimize clinical decision on RLE treatment.

Compared with the intracapsular method, the extracapsular method has the following advantages that are summarized from our clinical practice (11): (I) it reduces 
Table 3 Univariable and multivariable analysis for postoperative patients-reported outcome measures

\begin{tabular}{|c|c|c|c|c|c|c|c|}
\hline Score $(\mathrm{CR} / \mathrm{IR})$ & $\begin{array}{c}\text { Prognostic } \\
\text { factors }\end{array}$ & CR & IR & $\mathrm{t} / \chi^{2} / Z$ value & $P$ value & Adjusted OR (95\% Cl) & $\begin{array}{c}\text { Adjusted } \\
\text { P value }\end{array}$ \\
\hline \multirow[t]{5}{*}{ VAS (43/32) } & PVAS $^{a}$ & $6.1 \pm 2.0$ & $6.7 \pm 1.6$ & $t=-1.045$ & 0.3 & $0.823(0.614-1.103)$ & 0.193 \\
\hline & $\mathrm{Age}^{\mathrm{a}}$ & $44.0 \pm 6.5$ & $47.4 \pm 5.8$ & $t=-2.327$ & 0.023 & $0.914(0.842-0.993)$ & 0.033 \\
\hline & $N G l^{b}$ & $3.0(1.0,4.0)$ & $2.0(1.0,3.0)$ & $Z=-0.641$ & 0.521 & $0.998(0.809-1.231)$ & 0.987 \\
\hline & Suture $^{c}$ & $20(46.5 \%)$ & $14(43.8 \%)$ & $\chi^{2}=0.056$ & 0.812 & $1.426(0.484-4.204)$ & 0.52 \\
\hline & $\mathrm{PCF}^{\mathrm{c}}$ & $15(34.9 \%)$ & $6(18.8 \%)$ & $\chi^{2}=2.369$ & 0.124 & $0.437(0.100-1.910)$ & 0.271 \\
\hline \multirow[t]{4}{*}{ Mayo $(55 / 20)$} & PMEPS $^{\mathrm{a}}$ & $58.2 \pm 18.1$ & $55.7 \pm 20.3$ & $t=0.509$ & 0.613 & $0.999(0.969-1.031)$ & 0.961 \\
\hline & $\mathrm{MF}^{\mathrm{d}}$ & $11(20.0 \%)$ & $2(10.0 \%)$ & $\chi^{2}=0.445$ & 0.505 & $0.364(0.043-3.041)$ & 0.35 \\
\hline & Suture $^{c}$ & $25(45.5 \%)$ & $9(45.0 \%)$ & $\chi^{2}=0.001$ & 0.972 & $1.223(0.392-3.820)$ & 0.728 \\
\hline & $\mathrm{PCF}^{\mathrm{c}}$ & $16(29.1 \%)$ & $5(25.0 \%)$ & $\chi^{2}=0.122$ & 0.727 & $1.203(0.260-5.573)$ & 0.814 \\
\hline \multirow[t]{4}{*}{ DASH (32/43) } & $\mathrm{PDASH}^{\mathrm{a}}$ & $46.0 \pm 25.9$ & $50.7 \pm 24.4$ & $t=-0.799$ & 0.427 & $1.003(0.980-1.025)$ & 0.822 \\
\hline & $\operatorname{Sex}^{\mathrm{c}}$ & $16(50.0 \%)$ & $16(37.2 \%)$ & $\chi^{2}=1.227$ & 0.268 & $2.228(0.716-6.931)$ & 0.167 \\
\hline & $\mathrm{Age}^{\mathrm{a}}$ & $43.9 \pm 6.5$ & $46.6 \pm 6.1$ & $\mathrm{t}=-1.819$ & 0.073 & $0.945(0.871-1.025)$ & 0.17 \\
\hline & $\mathrm{NGl}^{\mathrm{b}}$ & $3.0(1.0,4.0)$ & $2.0(1.0,3.0)$ & $Z=-0.897$ & 0.37 & $1.187(0.958-1.470)$ & 0.117 \\
\hline
\end{tabular}

Test of normality for continuous variables. The distributions of age, PVAS, PMEPS and PDASH are normal and described as mean standard deviation, and the independent sample t test was used to compare the variables between different outcomes (a); NGI are not normally distributed and are described by median and quartile, and the Mann-Whitney $U$ test was used to compare the differences of PVAS between different outcomes (b). For the two-category variables, cases without microfracture, suture, or plaster cast fixation are the reference groups, and the proportion of patients with these is provided. When comparing two categorical variables between two outcomes, the Pearson $\chi^{2}$ test (c) or the continuous correction $\chi^{2}$ test (d) was adopted according to the expected value of the four-fold table. CR, complete recovery; IR, incomplete recovery; PVAS, preoperative VAS; PMEPS, preoperative MEPS; PDASH, preoperative DASH; NGI, number of glucocorticoid injections; MF, microfracture; PCF, plaster cast fixation.

the risk of joint fluid leakage and neurovascular injury in the joint; (II) the supine position saves time and effort, and the direct view of the CEO makes operations such as debridement, microfracture, and tear suture easier; and (III) it is relatively simpler than the intracapsular method, and the learning curve is flat, which makes it convenient for newer doctors to learn and promote. However, as a new method for the treatment of RLE, no well-accepted operative specification for extracapsular method was reported, and thus limits the popularization, standardization and quality control of it. Case-control trials or randomized trials are needed to investigate the clinical effect of extracapsular method in comparison with other methods.

\section{Technical and rehabilitation factors that affect prognosis}

Two main techniques are used to treat pathological tendon tissue in lateral epicondylalgia surgery: debridement and 
release. Debridement was adopted in this study. Solheim et al. (2) conducted a retrospective study of the techniques, and the results demonstrated that both are effective in the treatment of the diseased tissue and obtain satisfactory results. Compared with tendon release, the operation time for debridement is shorter, the cost of surgery is lower, trauma is reduced, and loss of muscle strength is smaller. However, randomized controlled trials are required to compare the techniques.

In this study, on the basis of debridement, we also performed microfractures on the cortical bone of the lateral epicondyle in 13 cases. Microfracture or drilling has been used in previous studies to activate repair mechanisms $(10,23)$. Yan et al. (10) argued that microfracture can accelerate recovery to physical exercise and increase surgery satisfaction; however, Khasaba (24) suggested that microfracture or drilling increased damage to the body, causing prolonged pain time, increased bleeding, and poor mobility. As a bone marrow stimulation technique, microfracture can activate bone marrow mesenchymal stem cells and promote the generation of bone, cartilage, and fibrous connective tissue; it is widely used in knee cartilage repair $(25,26)$. Compared with drilling, microfracture causes less trauma, fewer complications, and more reliable results. In this study, multivariate analysis indicated that treatment by microfracture was significantly associated with poor prognosis, which suggests that the body damage caused by microfracture outweighs its benefits. However, microfracture was only performed on patients whose cortical bone of the tendon attachment point is completely exposed after debridement, which indicates that these patients may be in more severe condition before surgery. This result requires confirmation by further research.

The extracapsular method allows the surgeon to look at the surface of the extensor tendon directly from the outside, making it possible to quickly suture the tendon tear under the endoscope. Yan et al. (10) sutured most identified CEO tendon tears, and the results suggested that suture had no significant effect, which our results confirmed. However, we performed endoscopic suture only in cases with tears after debridement, and those without tears were not sutured. Therefore, tear suture may improve the prognosis of patients with tears in the tendons. Whether tear suture contributes to the improvement of prognosis remains questionable, and further clinical randomized controlled studies are required to confirm its effectiveness.

For postoperative rehabilitation, most authors suggest that passive elbow joint activity training can be conducted after pain relief following surgery $(1,27)$, and active exercise should be performed as soon as possible (16). Some, however, argue that a period of rest after surgery is required before normal activities should be permitted $(2,28)$. For the first 21 cases, we used plaster cast fixation for 2 weeks, and (9.5\%) periarthritis symptoms appeared in 2 cases. We considered that this outcome was related to the limitation of shoulder joint movement caused by plaster fixation, and plaster cast fixation was not used in later cases. Among the results of multivariate analysis, plaster fixation was associated with a good prognosis regarding DASH score, suggesting that a period of postoperative movement restriction is conducive to the recovery of pain and function. However, due to the relatively high probability of periarthritis of the shoulder, whether plaster should be used may be a question that requires further large-scale prospective research.

\section{Demographic factors that affect prognosis}

The relationship between age and prognosis in lateral epicondylalgia surgical treatment is controversial. Solheim et al. (12) suggested that the recovery of function in younger individuals was poor; Yoon et al. (13) concluded the opposite. The results of Simon et al. (29) demonstrated a correlation between advanced age and postoperative pain caused by exercise. The slow recovery of pain in elderly patients may be related to age-related imbalance between endogenous excitatory and inhibitory processes in the neural system, and older adults are in a state of sustained pain stimulation (30). In our study, a correlation between advanced age and poor pain prognosis was identified. The relationship between age and postoperative pain recovery is another area requiring further study.

The results of the present study differed from those our previous studies (11), which suggested that females have poor prognosis compared with males. This may relate to the sample sizes. In this study, the sample was larger and more variables were available in the multivariable model, making the present results more robust.

The main limitation of this study is the lack of a control group, which may produce bias in statistical results. The selection of different surgical techniques among patients is based on indications and certain condition of different patients instead of randomly allocating among cases, thus introduces confounding factors and affects the statistical 
efficacy. Randomized controlled trials are required to clarify effects. Furthermore, although all patients were followedup for more than 12 months, follow-up time still varied widely among patients (12 to 70 months). The difference among follow-up time may have an effect on the results of this study.

\section{Conclusions}

To sum up, extracapsular endoscopic treatment of RLE has satisfactory and stable clinical results and high safety and learnability. The main technical points include debridement, microfracture, and tendon suture under the endoscope, all of which are easy to master and perform. However, old patients tended to have poor clinical results. Postoperative plaster fixation may help improve the prognosis of extracapsular endoscopic treatment, while microfracture may not be helpful to the prognosis.

\section{Acknowledgments}

Funding: This study was funded by National Key Research and Development Program of China (2018YFF0301100 and 2019YFF0302305). The funders had no role in the study design, data collection and analysis, decision to publish, or preparation of the manuscript.

\section{Footnote}

Reporting Checklist: The authors have completed the STROBE reporting checklist. Available at http://dx.doi. org/10.21037/atm-20-6799

Data Sharing Statement: Available at http://dx.doi. org/10.21037/atm-20-6799

Conflicts of Interest: All authors have completed the ICMJE uniform disclosure form (available at http://dx.doi. org/10.21037/atm-20-6799). The authors have no conflicts of interest to declare.

Ethical Statement: The authors are accountable for all aspects of the work in ensuring that questions related to the accuracy or integrity of any part of the work are appropriately investigated and resolved. The study was conducted in accordance with the Declaration of Helsinki (as revised in 2013). The study was approved by Peking University Third Hospital Ethics Review Board (NO.:
2018-219-011) and informed consent was taken from all individual participants.

Open Access Statement: This is an Open Access article distributed in accordance with the Creative Commons Attribution-NonCommercial-NoDerivs 4.0 International License (CC BY-NC-ND 4.0), which permits the noncommercial replication and distribution of the article with the strict proviso that no changes or edits are made and the original work is properly cited (including links to both the formal publication through the relevant DOI and the license). See: https://creativecommons.org/licenses/by-nc-nd/4.0/.

\section{References}

1. Oki G, Iba K, Sasaki K, et al. Time to functional recovery after arthroscopic surgery for tennis elbow. J Shoulder Elbow Surg 2014;23:1527-31.

2. Solheim E, Hegna J, Oyen J. Arthroscopic versus open tennis elbow release: 3- to 6-year results of a case-control series of 305 elbows. Arthroscopy 2013;29:854-9.

3. Pierce TP, Issa K, Gilbert BT, et al. A Systematic Review of Tennis Elbow Surgery: Open Versus Arthroscopic Versus Percutaneous Release of the Common Extensor Origin. Arthroscopy 2017;33:1260-8.e2.

4. Pomerantz ML. Complications of lateral epicondylar release. Orthop Clin North Am 2016;47:445-69.

5. Cohen MS, Romeo AA, Hennigan SP, et al. Lateral epicondylitis: anatomic relationships of the extensor tendon origins and implications for arthroscopic treatment. J Shoulder Elbow Surg 2008;17:954-60.

6. Cohen MS, Romeo AA. Open and arthroscopic management of lateral epicondylitis in the athlete. Hand Clin 2009;25:331-8.

7. Brooks-Hill AL, Regan WD. Extra-articular arthroscopic lateral elbow release. Arthroscopy 2008;24:483-5.

8. Rubenthaler F, Wiese M, Senge A, et al. Long-term follow-up of open and endoscopic Hohmann procedures for lateral epicondylitis. Arthroscopy 2005;21:684-90.

9. Grifka J, Boenke S, Krämer J. Endoscopic therapy in epicondylitis radialis humeri. Arthroscopy 1995;11:743-8.

10. Yan H, Cui GQ, Liu YL, et al. A randomized comparison of open and arthroscopic Nirschl debridement for refractory lateral epicondylitis. Zhonghua Wai Ke Za Zhi 2009;47:888-91.

11. Yang YP, Yuan S, Li N, et al. Analysis of factors influencing the efficacy of extracapsular arthroscopic surgery for refractory tennis elbow. Zhonghua wai ke za zhi 2019;57:818-23. 


\section{Page 10 of 10}

12. Solheim E, Hegna J, Oyen J. Extensor tendon release in tennis elbow: results and prognostic factors in 80 elbows. Knee Surg Sports Traumatol Arthrosc 2011;19:1023-7.

13. Yoon JP, Chung SW, Yi JH, et al. Prognostic Factors of Arthroscopic Extensor Carpi Radialis Brevis Release for Lateral Epicondylitis. Arthroscopy 2015;31:1232-7.

14. Solheim E, Hegna J, Oyen J, et al. Arthroscopic Treatment of Lateral Epicondylitis: Tenotomy Versus Debridement. Arthroscopy 2016;32:578-85.

15. Budoff JE, Hicks JM, Ayala G, et al. The reliability of the "Scratch test". J Hand Surg Eur Vol 2008;33:166-9.

16. Szabo SJ, Savoie FH, 3rd, Field LD, et al. Tendinosis of the extensor carpi radialis brevis: an evaluation of three methods of operative treatment. J Shoulder Elbow Surg 2006; 15:721-7.

17. Longo UG, Franceschi F, Loppini M, et al. Rating systems for evaluation of the elbow. Br Med Bull 2008;87:131-61.

18. Celik D. Psychometric properties of the Mayo Elbow Performance Score. Rheumatol Int 2015;35:1015-20.

19. Gay RE, Amadio PC, Johnson JC. Comparative responsiveness of the disabilities of the arm, shoulder, and hand, the carpal tunnel questionnaire, and the SF-36 to clinical change after carpal tunnel release. J Hand Surg Am 2003;28:250-4.

20. Mintken PE, Glynn P, Cleland JA. Psychometric properties of the shortened disabilities of the Arm, Shoulder, and Hand Questionnaire (QuickDASH) and Numeric Pain Rating Scale in patients with shoulder pain. J Shoulder Elbow Surg 2009;18:920-6.

21. Walz DM, Newman JS, Konin GP, et al. Epicondylitis:

Cite this article as: Yang YP, Yuan S, Cui GQ, An N, Ao YF. Extracapsular subcutaneous endoscopic treatment for refractory lateral epicondylalgia: technique, retrospective results, and prognostic factors. Ann Transl Med 2021;9(9):782. doi: 10.21037/atm-20-6799
Yang et al. Extracapsular method for refractory lateral epicondylalgia

pathogenesis, imaging, and treatment. Radiographics 2010;30:167-84.

22. Clark T, McRae S, Leiter J, et al. Arthroscopic Versus Open Lateral Release for the Treatment of Lateral Epicondylitis: A Prospective Randomized Controlled Trial. Arthroscopy 2018;34:3177-84.

23. Dunn JH, Kim JJ, Davis L, et al. Ten- to 14-year follow-up of the Nirschl surgical technique for lateral epicondylitis. Am J Sports Med 2008;36:261-6.

24. Khashaba A. Nirschl tennis elbow release with or without drilling. Br J Sports Med 2001;35:200-1.

25. Ahmad Z, Wardale J, Brooks R, et al. Exploring the application of stem cells in tendon repair and regeneration. Arthroscopy 2012;28:1018-29.

26. Gobbi A, Karnatzikos G, Kumar A. Long-term results after microfracture treatment for full-thickness knee chondral lesions in athletes. Knee Surg Sports Traumatol Arthrosc 2014;22:1986-96.

27. Terra BB, Rodrigues LM, Nahssen Filho A, et al. Arthroscopic treatment for chronic lateral epicondylitis. Rev Bras Ortop 2015;50:395-402.

28. Wada T, Moriya T, Iba K, et al. Functional outcomes after arthroscopic treatment of lateral epicondylitis. J Orthop Sci 2009; 14:167-74.

29. Simon CB, Riley JL, 3rd, Coronado RA, et al. Older Age as a Prognostic Factor of Attenuated Pain Recovery After Shoulder Arthroscopy. PM R 2016;8:297-304.

30. Lautenbacher S. Experimental approaches in the study of pain in the elderly. Pain Med 2012;13 Suppl 2:S44-50. 